\title{
Fungal infections of the central nervous system: clinical, radiographic and laboratory manifestations
}

\begin{abstract}
Objective: To assess the epidemiological aspects of central nervous system (CNS) fungal infections at 2 tertiary care teaching hospitals.

Patients and methods: We conducted a retrospective electronic medical record review of clinical, radiographic, laboratory, and microbiological data from patients at Mayo Clinic (Rochester, MN and Jacksonville, FL) with a proven or probable diagnosis of a CNS fungal infection from January 1, 2000 to December 31, 2014.

Results: We identified 97 (91 proven, 6 probable) CNS fungal infections; 73 had fungal meningitis, 18 had a tissue diagnosis, and 6 had CNS imaging indicative of infection with positive fungal cultures from other organs. Most patients $(67 \%)$ were male with a median age of 60 years; $64 \%$ were immunosuppressed, $29 \%$ of whom received prednisone $\geq 20 \mathrm{mg}$ daily for $\geq 2$ weeks. Cryptococcus spp was the most frequent infection identified (57\%) and headache $(47 \%)$ was the most common presenting symptom. Survival was significantly worse for patients with CNS aspergillosis compared to those with CNS Cryptococcus spp (hazard ratio, $10.92 ; P<0.001$ ).
\end{abstract}

Conclusion: CNS fungal infections have increased in frequency, particularly in patients requiring immunosuppression; most infections are caused by Cryptococcus spp. Aspergillus spp. CNS infections had 100\% mortality. High-dose prednisone was the most common cause of immunosuppression, and headache was the most frequent symptom at presentation.

Keywords: central nervous system, systemic fungal infection, immunocompromised state, epidemiology
Volume 5 Issue 6 - 2017

Lisa M Brumble,' Mohammed B Reza,' Laxmi P Dhakal, ${ }^{2,5,8}$ Grace Cruz, ${ }^{3}$ Omar MAbu Saleh,' Michael G Heckman, ${ }^{6}$ Emily R Vargas, ${ }^{6}$ Dennis W Dickson, ${ }^{7}$ Mark J Enzler,' Claudia R Libertin,' 'William D Freeman $2,4,5$

'Department of Infectious Disease, Mayo Clinic, USA

${ }^{2}$ Department of Neurology, Mayo Clinic, USA

${ }^{3}$ Department of Anesthesiology, Mayo Clinic, USA ${ }^{4}$ Department of Neurosurgery, Mayo Clinic, USA

${ }^{5}$ Department of Critical Care, Mayo Clinic, USA

${ }^{6}$ Department of Health Sciences Research, Mayo Clinic, USA

${ }^{7}$ Division of Anatomic Pathology, Mayo Clinic, USA

${ }^{8}$ Neurocritical Care Unit, Wesley Medical Center, USA

Correspondence: Lisa Brumble, Mayo Clinic, Division of Infectious Diseases, 4500 San Pablo Road, Jacksonville, FL 32224, USA, Tel 904-953-2419, Fax 904-953-0017,

Email Brumble.Lisa@mayo.edu

Received: September 08, 2017 | Published: October 03, 2017
Abbreviations: CI, confidence interval; CNS, central nervous system; CSF, cerebral spinal fluid; CT, computed tomography; HR, hazard ratio; MRI, magnetic resonance imaging

\section{Introduction}

Due to advancements in transplantation, the concomitant use of immunosuppressive therapies, and the pandemic spread of HIV, the incidence of systemic fungal infections, especially life-threatening central nervous system (CNS) infections, has increased. ${ }^{1}$ CNS fungal infections are associated with high mortality and morbidity. Even in immunocompetent hosts, the cure rate for patients receiving antifungal therapy for cryptococcal meningitis is $75 \%$ and is only $25 \%$ for CNS aspergillosis and mucormycosis. ${ }^{2}$

CNS fungal infections can be categorized as parenchymal (granulomas, cerebritis, abscess), extra-axial (meningitis), or vascular (vasculitis). ${ }^{3}$ Fungi are ubiquitous in the environment, and CNS infection often occurs after primary pulmonary infection. Delay in diagnosis of fungal infections is a major complicating factor. Symptoms are nonspecific, and even patients with disseminated fungal infection with multiorgan involvement may not present with organ-specific changes or clinical signs. ${ }^{4}$ Neurologic complications develop in half of all patients diagnosed with a fungal CNS infection. Accurate epidemiologic and prevalence data are not available because CNS fungal infections are not deemed by law as reportable diseases. ${ }^{1,2}$

CNS fungal infections carry a higher risk of morbidity and mortality compared to other infections and, therefore, require prompt diagnosis and appropriate medical and surgical management to optimize their outcomes.1 Given the limited data on the epidemiological aspects of CNS fungal infections, we conducted a retrospective electronic medical record review of CNS fungal infections at two Mayo Clinic sites (Rochester and Jacksonville).

\section{Materials and methods}

\section{Study design}

After institutional review board approval, we conducted a retrospective review of a consecutive series of patients with proven or probable cases of CNS fungal infection who were treated at Mayo Clinic in Jacksonville, FL, and Rochester, MN, from January 1, 2000 to December 31, 2014.

Patients were identified by review of the microbiology and pathology databases at each institution by searching for positive serology; fungal culture; biopsy results from CNS specimens, including brain, meninges, and $\mathrm{CSF} /$ ventricular fluid; and any confirmatory autopsy results. Any subsequent CNS fungal infection in the same patient with the same organism was counted as a single case. All cases of CNS infections identified via this process were confirmed and coded as proven or probable by detailed chart review.

\section{Case definitions}

We utilized the European Organization for Research and Treatment of Cancer/Invasive Fungal Infectious Cooperative Group (EORTC/ MSG) definition of invasive fungal disease, which categorized invasive fungal disease as a proven or probable infection. ${ }^{5}$ Proven cases were defined as those with a positive CNS tissue biopsy or CSF culture via lumbar puncture or ventricular fluid, as well as cases in which a positive fungal antigen or PCR test was obtained in the 
presence of active clinical signs of an infection (fever, headache, nuchal rigidity, or altered mental status) or focal neurologic deficits including seizures or stroke-like symptoms.

Probable cases included patients with host factors for immunosuppression and focal lesions on imaging or meningeal enhancement on magnetic resonance imaging (MRI) or computed tomography (CT) who met one or more additional mycological criteria, defined as:

i. Positive direct test (cytology, direct microscopy, or cultures) including presence of fungal elements or culture of mold in bronchial alveolar lavage fluid, sputum, or blood or

ii. Positive indirect test including antigen or fungal elements detected in plasma, serum, bronchial alveolar lavage fluid, or urine.

\section{Case data}

Fungal infection data was obtained by searching Misys and SoftMic databases in Rochester and ProNet in Jacksonville. Autopsy data was obtained from respective institution pathology departments. The day of diagnosis of the invasive fungal infection was the day on which the first diagnostic culture or examination was found to be positive. For each proven or probable case, a detailed chart review was performed to identify patient characteristics including demographic data, signs and symptoms at presentation, immunosuppressed states (defined as a patient maintained on immunosuppressive therapies after solid organ or bone marrow transplant, AIDS with CD 4 count $<200$ cells $/ \mu \mathrm{L}$, chemotherapeutic agent use, high-dose steroids of $\geq 20 \mathrm{mg}$ prednisone daily for $\geq 2$ weeks, use of immunomodulators for other indications, and leukopenia). Risk factors for CNS fungal infection were also evaluated, including the presence of CNS hardware or recent CNS surgery, end-stage renal or hepatic disease, diabetes mellitus, total parenteral nutrition use, intravenous drug abuse, head trauma, residence in endemic areas, and long-term central line use. ${ }^{6}$

\section{Statistical analysis}

Continuous variables were summarized using the sample median and range. Categorical variables were summarized using number and percentage of patients. The Kaplan-Meier method was used to estimate patient survival after diagnosis of CNS fungal infection, where censoring occurred on the date of last follow-up. Unadjusted Cox proportional hazards regression models were used to evaluate the associations of organism type and immunosuppression type with survival after diagnosis of CNS fungal infection. Hazard ratios (HRs) and $95 \%$ confidence intervals (CIs) were estimated. P-values of 0.05 or lower were considered statistically significant. All statistical analysis was performed using SAS (version 9.2; SAS Institute, Inc., Cary, North Carolina).

\section{Results}

During the study period, there were 91 proven and 6 probable CNS fungal infections identified for a total of 97 patients who met the criteria defined above and were selected for inclusion in this study. Seventy-four patients were identified in Rochester, MN and 23 in Jacksonville, FL. Diagnoses of CNS fungal infection included 73 with CSF antigen or cultures positive for fungal meningitis, 18 with tissue diagnosis, and 6 with CNS imaging indicative of an infection with positive fungal cultures from other organs (eg, blood, sputum, or urine).
Patient demographics are provided in Table 1. The median age was 60 years, and 65 patients $(67 \%)$ were male. Most patients $(75 \%)$ were white. Sixty-two patients (64\%) were immunosuppressed, and 33 patients (32\%) had risk factors for CNS fungal infection. Four patients (3\%) were neither immunosuppressed nor had risk factors for CNS fungal infections. Cryptococcus spp was the cause of CNS infection in all 4 healthy adults. The most common immunosuppressant used was high-dose prednisone $\geq 20 \mathrm{mg}$ daily for $\geq 2$ weeks for 18 patients (24\%) (Table 1).

Table I Demographics of 97 Patients CNS Fungal Infection

\begin{tabular}{|c|c|}
\hline Variable & Summary $(\mathrm{N}=97)$ \\
\hline Age & $60(18,87)$ \\
\hline Male & $57(18,87)$ \\
\hline Female & $60(25,83)$ \\
\hline \multicolumn{2}{|l|}{ Gender } \\
\hline Male & $65(67.0 \%)$ \\
\hline Female & 32 (33.0\%) \\
\hline \multicolumn{2}{|l|}{ Race } \\
\hline White & 73 (75.3\%) \\
\hline Unknown & II (II.3\%) \\
\hline Black or African American & $6(6.2 \%)$ \\
\hline Asian & $4(4.1 \%)$ \\
\hline American Indian/Alaskan Native & $2(2.1 \%)$ \\
\hline Other & I (I.0\%) \\
\hline \multicolumn{2}{|l|}{ Immunosuppressed } \\
\hline Yes & $62(63.9 \%)$ \\
\hline No & $35(36.1 \%)$ \\
\hline \multicolumn{2}{|l|}{ Immunosuppressed Due To } \\
\hline Prednisone $\geq 20 \mathrm{mg}$ per day & I 8 (29.0\%) \\
\hline Immunomodulator use & $16(25.8 \%)$ \\
\hline Chemotherapeutic agent use & $12(19.4 \%)$ \\
\hline Tacrolimus & $12(19.4 \%)$ \\
\hline Mycophenolate & $12(19.4 \%)$ \\
\hline AIDS CD4<200 & $5(8.1 \%)$ \\
\hline Idiopathic lymphopenia & $4(6.5 \%)$ \\
\hline Cyclosporine & $2(3.2 \%)$ \\
\hline
\end{tabular}

As displayed in Table 2, Cryptococcus spp was the most common cause of CNS fungal infection, occurring in 55 patients (57\%). This was followed by Candida spp in 16 patients (17\%), Aspergillus in 6 patients $(6 \%)$, Blastomyces in 5 patients $(5 \%)$, Coccidiosis in 4 patients (4\%), Scedosporium in 3 patients (3\%), and other types in the remaining 8 patients $(8 \%)$ (Table 2). Headache was the most common presenting symptom, occurring in 46 patients (47\%). Other relatively common symptoms were stroke-like symptoms (26\%), fever $(25 \%)$, and generalized weakness and myalgia (17\%) (Table 3$)$.

Table 2 Fungal Organism Types

\begin{tabular}{ll}
\hline Organism type & No. (\%), (N=97) \\
\hline Cryptococcus & $55(56.7 \%)$ \\
Candida & $16(16.5 \%)$ \\
Aspergillus & $6(6.2 \%)$ \\
Blastomyces & $4(4.2 \%)$ \\
Coccidiosis & $4(4.1 \%)$ \\
Scedosporium & $3(3.1 \%)$ \\
Other & $8(8.3 \%)$ \\
\hline
\end{tabular}

The "Other" category includes patients with Cladophialophora bantiana, Cladosporium, Fusarium, Histoplasmosis, Phaeohyphomycosis, Pseudoallesceria boydii/secrosporidium, Trametes, and Zygomyces 
Table 3 Presenting Symptoms and Clinical Features

\begin{tabular}{ll}
\hline Symptom/Feature & No. (\%), (N=97) \\
\hline Headache & $46(47.4 \%)$ \\
Stroke like Symptoms & $25(25.8 \%)$ \\
Fever & $24(24.7 \%)$ \\
Generalized weakness and Myalgia & $16(16.5 \%)$ \\
Acute mental status changes & $12(12.4 \%)$ \\
Progressive confusion/Dementia & $11(11.3 \%)$ \\
Nausea or Vomiting & $10(10.3 \%)$ \\
Seizures & $7(7.2 \%)$ \\
Visual disturbances, Loss of vision, Photophobia & $5(5.2 \%)$ \\
Syncope & $3(3.1 \%)$ \\
Neck pain & $3(3.1 \%)$
\end{tabular}

With a median length of follow-up of 11.7 months after CNS fungal infection diagnosis (range, 0 days to 147.1 months), a total of 48 patients $(49 \%)$ died. There was a significant difference in mortality between various fungi $(\mathrm{P}=0.002)$. For Cryptococcus, the most commonly diagnosed organism, 24 of 55 patients (44\%) died, and all 6 patients with Aspergillus died (HR, 10.92; 95\% CI, 4.07-29.30; $\mathrm{P}<0.001)$. There were no other significant differences in mortality between other fungi compared to Cryptococcus (all $\mathrm{P} \geq 0.10$ ). In the 62 immunosuppressed patients, mortality was significantly lower in patients who received mycophenolate mofetil compared to other patients (3/12 deaths $(25 \%)$; HR, $0.29 ; 95 \%$ CI, 0.09 $0.97 ; \mathrm{P}=0.045)$. No other significant associations between type of immunosuppression and survival were noted (all $\mathrm{P} \geq 0.11$ ). High-dose steroids or immunomodulating medications were the most common immunosuppressive medications $(30 / 60,50 \%)$ identified in patients diagnosed with cryptococcal meningitis. Nine of 16 patients $(56 \%)$ diagnosed with CNS Candida spp infection had a CSF shunt in place prior to infection.

\section{Discussion}

There appears to be an increasing incidence of invasive fungal infections from $6.6 \%$ from 1993 to 1996 to $10.4 \%$ from 2001 to 2005 . This increase in frequency in the past two decades is likely attributed to multiple factors including an aging population, increased use of disease-modifying drugs for treatment of autoimmune disorders, malignancies requiring use of cytotoxic drugs, increased number of bone marrow and solid-organ transplantations requiring long-term use of immunosuppressive agents, and finally, the pandemic spread of HIV/AIDS. ${ }^{1,8}$

Clinical presentation of CNS fungal infection varies from meningitis, meningoencephalitis, stroke, cerebritis, and vasculitis to venous sinus thrombosis. ${ }^{1,9}$ CNS fungal infection is difficult to diagnose because of the paucity of laboratory tests for it and its nonspecific imaging and clinical features. The low rate of fungal isolation from CSF specimens can also confound the diagnostic accuracy in these patients. 2 Clinical features of fungal meningitis and meningoencephalitis are usually reported as headaches, fever, nausea, vomiting, visual impairment, papilledema, seizure, and acute mental status changes. ${ }^{1}$ Similar findings were noted in our patient population, with headache being the most common initial clinical presentation. Patients often presented with more than one complaint, not always localized to the CNS. Cerebral vascular accident or strokelike symptoms such as limb or facial weakness, slurred speech, gait abnormality, or ataxia were collectively the second most common clinical presentation in our patient population. One of the cases was a 68 year old female with high-grade myeloproliferative disorder with acute myeloid leukemia who presented with acute stroke symptoms of hemiparesis, followed by respiratory failure, and stupor. Initial imaging with noncontrast head CT (Figure 1A) showed no acute hypodensity. Subsequent MRI imaging (Figure 1B-1E) showed progressive vertebrobasilar vasculitic infarction of the right pons followed 3 days later by bilateral posterior circulation infarction. LP fluid was negative for fungal organism but autopsy was definitive of fungal progressive invasion of the basilar artery and circulation (Figure 2A-2E).

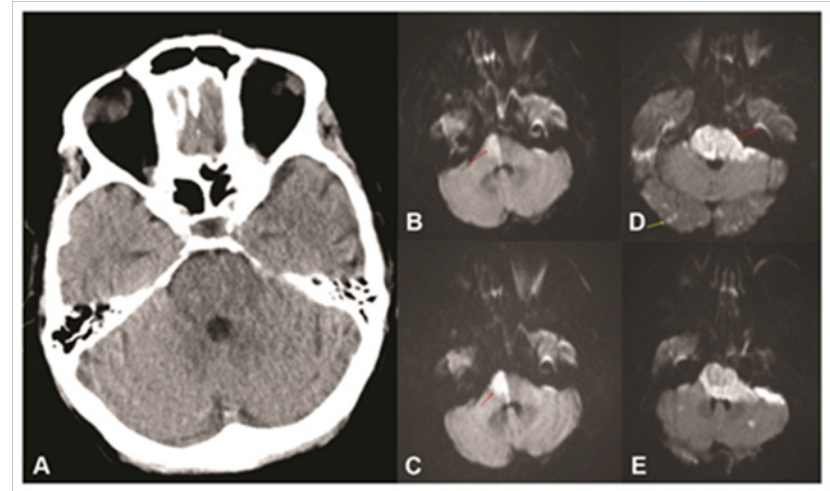

Figure I

(A) CT head without contrast at onset of hemiparesis which appears negative for bleeding or obvious infarction.

(B \& C) MRI DWI sequence showing right upper pontine ischemic changes (C higher cut).

(D \& E) demonstrate 3 days later after patient progressed to coma and impaired brainstem reflexes widespread basilar artery territory infarction and scattered diffuse changes in occipital lobes (peripheral) arrow in E which was a higher slice than $\mathrm{D}$.
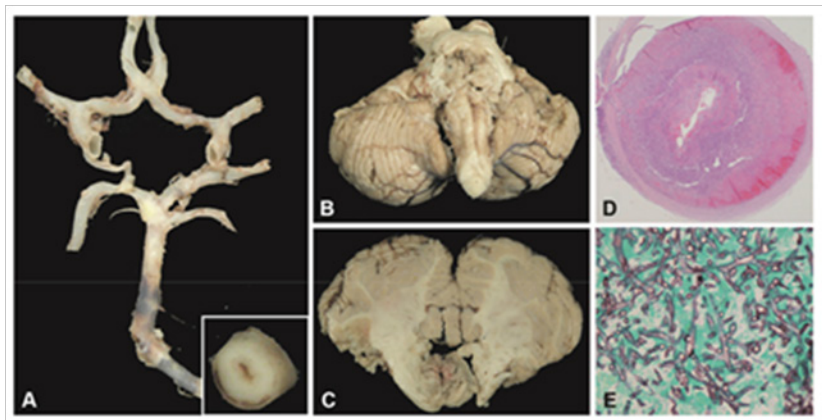

Figure 2

(A) Dissected circle of Willis, with thrombosis of basilar artery. (inset shows cross section of basilar artery with marked stenosis due to mural thickening.

(B) Ventral view of brainstem and cerebellum shows softening in the caudal pons and the left inferior cerebellar hemisphere. There is also tonsillar herniation.

(C) Transverse section of pons and cerebellum at level of middle cerebellar peduncle shows marked softening of the pontine base and chronic softening of the left inferior cerebellar hemisphere.

(D) Cross section of the basilar artery stained with hematoxylin and eosin shows mural thickening due to infiltrative inflammatory process and hemorrhage.

(E) Gomori silver stain for fungi reveals nonseptate fungi with variable width and orthogonal branching consistent with phycomycetes (invasive mucormycosis).

Previous studies have shown that Cryptococcus is the most common CNS fungal infection; it is rare in immunocompetent individuals. Pyrgos et al. ${ }^{10}$ published on the incidence and trends of 
cryptococcal meningitis-associated hospitalizations in 18 states from 1997 through 2009, 21.6\% of which occurred in patients who were HIV-negative. ${ }^{10}$ Cryptococcal meningitis in-hospital mortality was $12.4 \%$ for women and $10.8 \%$ for men. Risk factors for cryptococcal meningitis included solid organ or bone marrow transplantation, combined $\mathrm{T}$ and $\mathrm{B}$ cell defects, Cushing's syndrome, liver disease, and hypogammaglobulinemia. ${ }^{10}$ Similar findings were noted in our patient population, with 55 patients $(57 \%)$ with CNS fungal infection attributed to Cryptococcus. Of the 55 patients, 4 were healthy adults who were not on immunosuppressive medications and did not have any known risk factors for CNS fungal infections. Interestingly, all 4 patients were treated at Mayo Clinic in Rochester and lived in 4 different states in the Midwest.

Cryptococcus CNS infectionoccurs more frequently in patients with AIDS in Africa and Southeast Asia than in the United States. In developed countries, use of antiretroviral therapy has resulted in a reduction in the incidence of cryptococcal-related CNS infections. Cryptococcomas or brain mass lesions due to Cryptococcus neoformansare infrequent compared to meningitis. ${ }^{11}$ Similar findings were observed in our patient population; meningitis attributed to $C$. neoformans was the most common cause of CNS fungal infection in 9 of 10 patients with AIDS, with a median CD4 count of 34 cells $/ \mu \mathrm{L}$.

Reported cases of Candida CNS infection have increased, with reports ranging from $6 \%$ to $17 \%$ in neurosurgical patients. Factors such as immunocompromised state, prolonged use of broad-spectrum antibiotics and indwelling bladder and intravenous catheter use can increase the risk of Candida infection. ${ }^{12}$. Candida CNS infection can also occur in intravenous drug users and can present as meningitis, microabscesses with granuloma formation, frank abscess formation, or ependymitis. Candida is part of the normal intestinal flora, and infections usually occur following disturbances in the microbial equilibrium of the gut. ${ }^{2}$. Of the 16 cases of Candida CNS infection in our study, 9 (56\%) had CNS shunting in place prior to the infection, and $3(18 \%)$ had recently undergone neurosurgical procedures. Only $3(18 \%)$ were immunosuppressed at time of infection. Ten (62\%) of the 16 Candida CNS infections were due to Candia albicans, and 4 were due to Candida parapsilosis. Interestingly, all 4 patients with $C$. parapsilosis had CNS shunting prior to CNS infection.

A broad spectrum of immunosuppressed patients, including patients with hematologic malignancies, transplant recipients, and patients on chronic high-dose steroids are at risk for aspergillosis. In immunocompromised patients, $10 \%$ to $25 \%$ with pulmonary aspergillosis later developed CNS involvement. ${ }^{2}$. Spapen et al. ${ }^{13}$ reviewed a cohort of 563 adult patients with evidence of Aspergillus infection during their ICU stay. Ten patients with CNS aspergillosis were identified from the 563 patients. All had one or more host factors predisposing them to invasive aspergillosis. Aspergillus infection was confirmed by brain biopsy in 3 subjects, with a mortality rate of $90 \%{ }^{13}$

Further, in a case series of 14 immunosuppressed patients with CNS aspergillosis at Massachusetts General Hospital, 11 had aspergillosis focused in the lungs, 2 patients had paranasal sinus involvement, and 1 had spine discitis as the source of infection. ${ }^{14}$

CNS aspergillosis carries a high rate of mortality. Denning et al reviewed 4 case series 1,223 cases of invasive aspergillosis, and cerebral aspergillosis had a crude mortality rate of $99 \%{ }^{15}$ Similar findings were noted in our patient population. All 6 patients with $\mathrm{CNS}$ aspergillosis were on immunosuppressive medications, with high-dose long-term steroid use for 2 of 6 patients. CNS aspergillosis was diagnosed in 3 patients by tissue biopsy. Crude mortality rate at 1 -year post-CNS aspergillosis was $100 \%$. The lungs were the primary site of infection in 2 patients $(33 \%)$. One patient underwent living related kidney transplantation for treatment of endstage renal disease with brain CT scan performed 3 months posttransplant showing multiple ring enhancing lesions, biopsy of which grew Aspergillus spp. Two patients suffered $>1$ year of neutropenia due to myelodysplastic syndrome. Another patient presented with generalized weakness and diffuse upper extremity skin rash. Skin biopsy cultures yielded Aspergillus with CNS imagining showing multiple CNS lesions. The patient was receiving high-dose steroids and cyclophosphamide for management of necrotizing vasculitis.

Most cases of the endemic mycoses histoplasmosis, coccidioidomycosis, and blastomycosis occur in regions of the United States where these organisms are prevalent. Reports suggest that a significant proportion of these infections are due to recent exposure. ${ }^{16}$ In our patient population, 4 patients diagnosed with CNS blastomycosis infection were living in endemic states: Iowa, Minnesota, and Wisconsin. Only 1 of 4 patients was on immunosuppressive medication, receiving high-dose prednisone for treatment of polymyalgia rheumatica. All 4 patients presented with headaches. Pulmonary blastomycosis infection was the primary infection, with dissemination to the brain in all four cases.

Coccidioidomycosis (cocci) dissemination to the CNS occurs in less than $1 \%$ of patients with pulmonary coccidioidomycosis. Only $2 \%$ of patients with coccidioidal meningitis are immunocompromised. ${ }^{2}$ All 4 cases of CNS coccidioidomycosis in our patient population had lived in or recently visited Arizona. Two of the 4 patients were immunocompromised due to AIDS (CD4 count of 32) and ulcerative colitis flare. The patient was on high-dose of steroids and immunomodulating medication for treating ulcerative colitis flare. Three of the 4 patients had disseminated disease with positive cocci complement titers in serum. Mortality rate at 1 year with both cocci and blastomyces CNS infection was $25 \%$ in our patient population.

As we look forward to future development of new, ever more immunosuppressing therapies for management of autoimmune disorders and malignancies, this could further lead to an increased incidence of CNS fungal infections. CNS fungal infections may be unavoidable, but maintaining a high degree of clinical suspicion for immunocompromised patients is the first step to making the correct diagnosis. The importance of our study is that it provides an insight into the risk factors for CNS fungal infection, such as prolonged use of high-dose steroids, use of chemotherapeutic agents, and longterm immunosuppressant use. Patients with indwelling CSF shunts or who undergo CNS surgical procedures are at a high risk for CNS Candida infections. Our study findings are similar to prior reports showing Cryptococcus is the most common cause of CNS fungal infection, and we found a high rate of mortality in patients with CNS aspergillosis. An interesting finding was the presentation of strokelike symptoms as the second most common clinical presentation. Eid et al. ${ }^{17}$ reported a patient with CNS histoplasmosis who presented with slowly progressive weakness in the lower extremities followed by development of numbness below the midthoracic area, urinary incontinence, and slurred speech. Brain MRI revealed leptomeningeal enhancement with cerebral spinal fluid (CSF) fungal cultures eventually growing Histoplasma capsulatum. ${ }^{17}$ Stroke-like symptoms were the second most common clinical presentation in our patient population. Our study also highlights the fact that CNS fungal infection should be in the differential for patients presenting with slowly progressive neurological deficits. 
There were several important study limitations. The retrospective design of the study may have introduced bias in the data collection. Our cases were primarily referral cases and, therefore, are not representative of the local geographic area that we serve. We did not include a control group of patient who were immunosuppressed but did not experience CNS fungal infections. Although there is an association between high-dose prednisone use and CNS fungal infection, more research is necessary to determine if there is true causation. Alternatively, this may be attributed to the fact that prednisone is frequently prescribed by clinicians to treat a myriad of illnesses and may be a marker for severe illness, rather than being the causative factor predisposing patients to CNS fungal infections. Analysis of CSF by fungal culture, antigen studies, and PCR is insensitive in confirming the diagnosis of CNS fungal infections, making it difficult to capture all of the CNS fungal infections seen at our two centers during the study time period. There were 91 total patients with confirmed cases of CNS fungal infection (94\%) out of 97 patients included in the study. Given that the majority of patients included in this study had confirmed CNS fungal infections, the study gives us unique insight into their clinical presentation.

\section{Conclusion}

The study highlights the fact that clinicians caring for immunosuppressed patients should pay close attention to clinical presentations of headaches, seizures, or focal neurological deficits. Such presentations should trigger providers to consider CNS fungal infection. A high degree of suspicion and diagnostic acumen must be used to verify the diagnosis of CNS fungal infections in this patient population.

\section{Acknowledgements}

We thank Dustin Denmark for his excellent administrative assistance.

\section{Conflicts of interest}

The authors declare that they have no conflicts of interest.

\section{Funding}

No funding was obtained for this study.

\section{References}

1. Raman Sharma R. Fungal infections of the nervous system:Current perspective and controversies in management. Int J Surg. 2010;8(8):591601.

2. Perdigao J, Rojas R, Verzelli LF, et al. Fungal infections of the central nervous system. Semin Roentgenol. 2004;39(4):505-518.
3. Mathur M, Johnson CE, Sze G. Fungal infections of the central nervous system. Neuroimaging Clin N Am. 2012;22(4):609-632.

4. Liu X, Ling Z, Li L, Ruan B. Invasive fungal infections in liver transplantation. Int J Infect Dis. 2011;15(5):e298-e304.

5. De Pauw B, Walsh TJ, Donnelly JP, et al. Revised definitions of fungal disease from the European Organization for Research and Treatment of Cancer/Invasive Fungal Infections Cooperative Group and the National Institute of Allergy and Infectious Diseases Mycoses Study Group (EORTC/MSG) Consensus Group. Clin Infect Dis. 2008;46(12):18131821.

6. Sethi PK, Khanna L, Batra A, et al. Central nervous system fungal infections: Observations from a large tertiary hospital in northern India. Clin Neurol Neurosurg. 2012;114(9):1232-1237.

7. Lehrnbecher T, Frank C, Engels K, et al. Trends in the postmortem epidemiology of invasive fungal infections at a university hospital. $J$ Infect. 2010;61(3):259-265.

8. Chimelli L, Mahler Araujo MB. Fungal infections. Brain Pathol. 1997;7(1):613-627.

9. Black KE, Baden LR. Fungal infections of the CNS: treatment strategies for the immunocompromised patient. CNS Drugs. 2007;21(4):293-318.

10. Pyrgos V, Seitz AE, Steiner CA, et al. Epidemiology of cryptococcal meningitis in the US:1997-2009. PLoS One. 2013;8(2):e56269.

11. Zarrin M, Zarei Mahmoudabadi A. Central nervous system fungal infections; a review article. Jundishapur J Microbiol. 2010;3(2):41-47.

12. Montero A, Romero J, Vargas JA, et al. Candida infection of cerebrospinal fluid shunt devices:report of two cases and review of the literature. Acta Neurochir (Wien). 2000;142(1):67-74.

13. Spapen H, Spapen J, Taccone FS, et al. Cerebral aspergillosis in adult critically ill patients:a descriptive report of 10 patients from the AspICU cohort. Int J Antimicrob Agents. 2014;43(2):165-169.

14. Kourkoumpetis TK, Desalermos A, Muhammed M, et al. Central nervous system aspergillosis:a series of 14 cases from a general hospital and review of 123 cases from the literature. Medicine (Baltimore). 2012;91(6):328-336.

15. Denning DW. Therapeutic outcome in invasive aspergillosis. Clin Infect Dis. 1996;23(3):608-615.

16. Ampel NM. Emerging disease issues and fungal pathogens associated with HIV infection. Emerg Infect Dis. 1966;2(2):109-116.

17. Eid AJ, Leever JD, Husmann K. Compartmentalized Histoplasma capsulatum Infection of the Central Nervous system. Case Reports in Infectious Diseases. 2015:3. 